\title{
A New Framework for the Performance Analysis of Wireless Communications under Hoyt (Nakagami- $q$ ) Fading
}

\author{
Juan M. Romero-Jerez, Senior Member, IEEE, and F. Javier Lopez-Martinez, Member, IEEE
}

\begin{abstract}
We present a novel relationship between the distribution of circular and non-circular complex Gaussian random variables. Specifically, we show that the distribution of the squared norm of a non-circular complex Gaussian random variable, usually referred to as the squared Hoyt distribution, can be constructed from a conditional exponential distribution. From this fundamental connection we introduce a new approach, the Hoyt transform method, that allows to analyze the performance of a wireless link under Hoyt (Nakagami- $q$ ) fading in a very simple way. We illustrate that many performance metrics for Hoyt fading can be calculated by leveraging well-known results for Rayleigh fading and only performing a finite-range integral. We use this technique to obtain novel results for some information and communication-theoretic metrics in Hoyt fading channels.
\end{abstract}

Index Terms-Hoyt Fading, Rayleigh Fading, Rice $I e$-function, Channel Capacity, Performance Analysis, Secrecy Capacity.

\section{INTRODUCTION}

The characterization of the distribution of a complex Gaussian random variable (RV) is arguably one of the most relevant problems in engineering and statistics. In the contexts of information and communication theory, the distribution of the norm of the complex Gaussian random variable $Z=X+j Y$ (where $X$ and $Y$ are jointly Gaussian) finds application in many problems such as signal detection, noise characterization, or wireless fading channel modeling, just to name a few.

In the literature, the most general case ${ }^{1}$ of $X$ and $Y$ having

J. M. Romero-Jerez is with Departmento de Tecnología Electrónica, E.T.S.I. Telecomunicación, Universidad de Málaga, 29071 Málaga, Spain (e-mail romero@dte.uma.es).

F. J. Lopez-Martinez is with Departmento de Ingeniería de Comunicaciones, E.T.S.I. Telecomunicación, Universidad de Málaga, 29071 Málaga, Spain. (email: fjlopezm@ic.uma.es). During the elaboration of this paper, he was with Wireless Systems Lab, Electrical Engineering, Stanford University, Stanford, CA.

The work of Juan M. Romero-Jerez was supported by the Spanish Government-FEDER public Project No. TEC2013-42711-R. The work of F.J. Lopez-Martinez was funded by Junta de Andalucia (P11-TIC-7109), Spanish Government-FEDER (TEC2013-44442-P, COFUND2013-40259), the University of Malaga and the European Union under Marie-Curie COFUND U-mobility program (ref. 246550).

The material in this paper has been presented, in part, at the 2015 IEEE International Symposium on Information Theory and the 2015 European Wireless conference.

Copyright (c) 2017 IEEE. Personal use of this material is permitted. However, permission to use this material for any other purposes must be obtained from the IEEE by sending a request to pubs-permissions@ieee.org..

${ }^{1}$ Depending on the specific context, different authors have pursued the characterization of the envelope $E=\sqrt{X^{2}+Y^{2}}$ or the squared envelope $R=E^{2}$. However, both distributions are related by a simple transformation and therefore have a similar form. Throughout this paper, we will focus on the distribution of $R$, which models the instantaneous power of a complex Gaussian signal. different mean and variance was addressed by Beckmann [1], as a generalization of the previous results obtained by Rice [2] and Hoyt [3], and recently revisited in [4, 5]. In this general situation the chief distribution functions ( $\mathrm{pdf}$ and cdf) of $R=X^{2}+Y^{2}$ have complicated forms.

In the specific case of $X$ and $Y$ being independent Gaussian $\mathrm{RVs}$ with zero mean and arbitrary variance, or equivalently being correlated Gaussian RVs with zero mean and equal variances, the $\mathrm{RV} Z$ is said to be a zero mean non-circular (or improper) Gaussian RV [6]. This occurs in many practical scenarios, such as in the detection of non-stationary complex random signals [7], or in the characterization of multipath fading [8]. In this latter situation, the Hoyt [3] or Nakagami- $q$ fading [8] distribution is used to model short-term variations of radio signals resulting from the addition of scattered waves which can be described as a complex Gaussian RV where the in-phase and quadrature components have zero mean and different variances, or equivalently, where the in-phase and quadrature components are correlated. This distribution is commonly used to model signal fading due to strong ionospheric scintillation in satellite communications, or in general those fading conditions more severe than Rayleigh, and it includes both Rayleigh fading and one-sided Gaussian fading as special cases. Furthermore, it was shown in [9] that the second order statistics of Hoyt fading provide a best fit to field measurements in mobile satellite channels with heavy shadowing.

One of the main advantages of Rayleigh fading, which is perhaps the most popular model for the random fluctuations of the signal amplitude when transmitted through a wireless link when there is no direct line-of-sight (LOS) between the transmit and receive ends, relies on its comparatively simple analytic manipulation, as the received signal-to-noise ratio (SNR) is exponentially distributed. Conversely, the received SNR in Hoyt fading has a much more complicated form and sophisticated special functions are required to characterize the pdf or cdf [10] of a squared Hoyt RV.

Both Rayleigh and Hoyt fading have been extensively investigated in the last few decades [11]; however, while the derivation of information and communication-theoretic performance metrics such as channel capacity [12] and outage probability (OP) [13] is usually tractable mathematically for the Rayleigh case, it is way more complicated to analyze the same scenario when Hoyt fading is assumed.

Dozens of papers have been published in the last years with the aim of analyzing very diverse scenarios where Hoyt fading 
is considered, for the sake of extending already known results for Rayleigh fading to this more general situation [10, 1417]. However, despite the relationship that can be inferred between both distributions, existing analyses in the literature for Hoyt fading do not exploit this connection and usually require tedious and complicated derivations. To the best of our knowledge, there is no standard procedure that takes advantage of the relationship between both distributions, and therefore the calculations for Hoyt fading must be done from scratch.

In this paper, we present a novel connection between the distribution of the squared norm of a non-circular complex Gaussian RV and its circular counterpart. In other words, we introduce a useful relationship between the squared Hoyt distribution and the exponential distribution, which greatly simplifies the analysis of the former. By exploiting the fact that the cdf of a squared Hoyt distributed random variable is a weighted Rice $I e$-function, we demonstrate that the squared Hoyt distribution can be constructed from a conditional exponential distribution.

This connection has important relevance in practice: Since most communication-theoretic metrics are computed with a linear operation over the SNR distribution, we show that performance results for Hoyt fading channels can be readily obtained by leveraging previously known results for Rayleigh fading, and computing a simple finite-range integral. This general procedure will be referred to as the Hoyt transform method. The main takeaway is that there is no need to redo any calculation in order to analyze the performance of communication systems in Hoyt fading, if there are available results for the simpler Rayleigh case. Instead, the application of the Hoyt transform yields the desired performance result in a direct way.

In some cases, the Hoyt transform has analytical solution and hence the expressions for Hoyt fading are of similar complexity to those obtained for Rayleigh scenarios. Otherwise, the results for Hoyt fading have the form of a finite-range integral with constant integration limits, over the performance metric of interest for the Rayleigh case. Integrals of this form are quite common in communications, including properintegral forms for the Gaussian $Q$-function [18], the Marcum $Q$-function [19] or the Pawula $F$-function [20], or those obtained with the application of the moment generating function (MGF) approach to the calculation of error probability [21]. Therefore, the numerical computation of the Hoyt transform introduced in this paper is simpler than other alternatives that require the evaluation of infinite series or inverse Laplace transforms. As an additional advantage, our new approach also permits to obtain upper and lower bounds of different performance metrics in a simple way.

Using this general procedure, we provide novel analytical results for some scenarios of interest in information and communication theory; specifically:

- We analyze the Shannon capacity for adaptive rate transmission in Hoyt fading channels, thus extending the results given in [12]. Thanks to the Hoyt transform method, we can calculate the asymptotic capacity in the low and high-SNR regimes in closed-form. We show that the asymptotic capacity loss per bandwidth unit in the
high-SNR regime is up to $1.83 \mathrm{bps} / \mathrm{Hz}$ compared to the AWGN case, and up to $1 \mathrm{bps} / \mathrm{Hz}$ when compared to the Rayleigh case.

- We investigate the physical layer security of a wireless link in the presence of an eavesdropper, where both the desired and wiretap links are affected by Hoyt fading. Known analytical results are available for different scenarios such as Rayleigh [22], Nakagami-m [23], Rician [24], or Two-Wave with Diffuse Power [25] fading models. However, to the best of our knowledge, there are no results in the literature for the physical layer security in Hoyt fading channels.

The rest of this paper is organized as follows. In Section II, some preliminary definitions are introduced and the Rice $I e$-function is reviewed. Then, the main mathematical contributions are presented in Section III: the connection between the squared Hoyt and the exponential distributions, and its application to define the Hoyt transform method to the performance analysis in Hoyt fading channels. This approach is used in Sections IV and V to obtain analytical results in the aforementioned scenarios. Numerical results are given in Section VI, and the main conclusions are outlined in Section VII.

\section{DEFINITIONS AND PRELIMINARY RESULTS}

\section{A. Non-circular complex Gaussian RV}

Let $Z=X+j Y$ be a zero-mean non-circular complex Gaussian RV, where $X$ and $Y$ are independent jointly Gaussian RVs with variances $\sigma_{x}^{2}$ and $\sigma_{y}^{2}$. Then, the random variable $R=X^{2}+Y^{2}$ is said to follow the squared Hoyt distribution, and its pdf is given by

$$
f_{R}(x)=\frac{1+q^{2}}{2 q \bar{\gamma}} \exp \left[-\frac{\left(1+q^{2}\right)^{2} x}{4 q^{2} \bar{\gamma}}\right] I_{0}\left(\frac{\left(1-q^{4}\right) x}{4 q^{2} \bar{\gamma}}\right),
$$

where $I_{0}(\cdot)$ is the modified Bessel function of the first kind and zero order, $\bar{\gamma}=\mathbb{E}\{R\}$ and $q=\sigma_{y} / \sigma_{x}$ is the shape parameter, assuming without loss of generality that $\sigma_{y} \leq \sigma_{x}$. Therefore, we have $q \in[0,1]$.

If $q=1$, then $Z$ is a zero-mean circular complex Gaussian $\mathrm{RV}$ and therefore $R$ is exponentially distributed, with pdf $f_{R}(x)=\frac{1}{\gamma} e^{-x / \bar{\gamma}}$.

\section{B. The Rice Ie-function}

Let $k$ and $x$ be non-negative real numbers with $0 \leq k \leq 1$; then, the Rice $I e$-function is defined as [2]

$$
I e(k, x)=\int_{0}^{x} e^{-t} I_{0}(k t) d t .
$$

The Rice Ie-function admits different infinite series representations [26, 27], and it is not considered a tabulated function, in the sense that it is not included as a built-in function in standard mathematical software packages such as Matlab or Mathematica. However, after the appropriate change of notation this function can be written in compact form, as [28]

$$
I e(k, x)=\frac{1}{\sqrt{1-k^{2}}}[Q(\sqrt{a x}, \sqrt{b x})-Q(\sqrt{b x}, \sqrt{a x})]
$$


or equivalently,

$$
I e(k, x)=\frac{1}{\sqrt{1-k^{2}}}\left[2 Q(\sqrt{a x}, \sqrt{b x})-e^{-x} I_{0}(k x)-1\right],
$$

where $a=1+\sqrt{1-k^{2}}, b=1-\sqrt{1-k^{2}}$ and

$$
Q(\alpha, \beta)=\int_{\beta}^{\infty} t e^{-\frac{t^{2}+\alpha^{2}}{2}} I_{0}(\alpha t) d t
$$

is the first order Marcum $Q$-function.

Since both the modified Bessel function $I_{0}$ and the Marcum $Q$-function are tabulated functions, (3) and (4) can be easily computed. However, subsequent manipulations of these expressions are generally complicated and in many situations it may be preferable to express the Rice $I e$-function in integral form. Replacing $I_{0}(\cdot)$ in (2) by its integral representation, namely [29, eq. (8.431.5)]

$$
I_{0}(z)=\frac{1}{\pi} \int_{0}^{\pi} e^{z \cos \theta} d \theta,
$$

after some manipulation we can write [26]

$$
I e(k, x)=\frac{1}{\sqrt{1-k^{2}}}-\frac{1}{\pi} \int_{0}^{\pi} \frac{e^{-x(1-k \cos \theta)}}{1-k \cos \theta} d \theta,
$$

which has important advantages with respect to (2), as the integration limits do not depend on the arguments of the defined function, and the integrand is given in terms of elementary functions. However, for reasons that will become clear in the next Section, a much more convenient representation of the Rice $I e$-function for the purpose of this work is the one provided in the following proposition.

Proposition 1: The Rice Ie-function can be written in integral form as

$I e(k, x)=\frac{1}{\sqrt{1-k^{2}}}\left[1-\frac{1}{\pi} \int_{0}^{\pi} \exp \left(-x \frac{1-k^{2}}{1-k \cos \theta}\right) d \theta\right]$.

Proof: Although not specifically stated in this form, this result follows from the identities provided by Pawula in [28, eqs. (2a)-(2d)] [30, eqs. (7)-(10)]. In particular, from [30, eqs. (9)-(10)] the following identity holds:

$$
W \int_{0}^{\pi} \frac{e^{-(U-V \cos \theta)}}{U-V \cos \theta} d \theta=\int_{0}^{\pi} \exp \left(-\frac{W^{2}}{U-V \cos \theta}\right) d \theta
$$

where $W=\sqrt{U^{2}-V^{2}}$. By identifying $U=x$ and $V / U=k$, and with the help of (7), the desired expression is obtained.

\section{Main Results}

We now introduce the main mathematical contributions of this work, which are given in a set of lemmas and corollaries.

Lemma 1: Let $R \mid \theta$ be an exponentially distributed random variable, conditioned on $\theta$, with pdf

$$
f_{R \mid \theta}(x)=\frac{1}{\gamma(\theta, q)} e^{-x / \gamma(\theta, q)},
$$

where $\theta$ is a random variable uniformly distributed between 0 and $\pi$, and

$$
\gamma(\theta, q) \triangleq \bar{\gamma}\left(1-\frac{1-q^{2}}{1+q^{2}} \cos \theta\right)=\mathbb{E}\{R \mid \theta\} .
$$

Then, the unconditional random variable $R$, with pdf

$$
f_{R}(x)=\frac{1}{\pi} \int_{0}^{\pi} \frac{1}{\gamma(\theta, q)} e^{-x / \gamma(\theta, q)} d \theta,
$$

follows a squared Hoyt distribution with average $\mathbb{E}\{R\}=\bar{\gamma}$ and parameter $q$, i.e., (12) is an alternative expression for the pdf given in (1). The cdf of $R$ will be given by

$$
F_{R}(x)=1-\frac{1}{\pi} \int_{0}^{\pi} e^{-x / \gamma(\theta, q)} d \theta .
$$

Proof: The cdf of the $R$ can be calculated as

$F_{R}(x)=\int_{0}^{x} \frac{1+q^{2}}{2 q \bar{\gamma}} \exp \left[-\frac{\left(1+q^{2}\right)^{2} t}{4 q^{2} \bar{\gamma}}\right] I_{0}\left(\frac{\left(1-q^{4}\right) t}{4 q^{2} \bar{\gamma}}\right) d t$,

which can be written using the definition of the Rice Iefunction in (2) as

$$
F_{R}(x)=\frac{2 q}{1+q^{2}} \operatorname{Ie}\left(\frac{1-q^{2}}{1+q^{2}}, \frac{\left(1+q^{2}\right)^{2}}{4 q^{2} \bar{\gamma}} x\right) .
$$

Using the alternative definition for the Rice $I e$-function in (8), the cdf of the SNR can be written after some algebraic manipulation as in (13). Finally, by taking the derivative of (13), the desired pdf in (12) is obtained.

By comparing (12) with the pdf of an exponential distribution, Lemma 1 states that a squared Hoyt RV can be viewed as a finite-range integral of an exponentially distributed RV with continuously varying average. Note that the factor that multiplies $\cos \theta$ in (11) coincides with the squared third eccentricity $\epsilon$ of the ellipse represented by the underlying non-circular complex Gaussian random variable of the Hoyt distribution [31], i.e. $\epsilon=\frac{1-q^{2}}{1+q^{2}}$.

A direct application of Lemma 1 in a communicationtheoretic context follows: Any performance metric in Hoyt fading channels that can be obtained by averaging over the SNR pdf (e.g. outage probability, channel capacity, error probability) can be calculated from existing results for Rayleigh fading, by performing a finite-range integral. In this situation, $\bar{\gamma}$ represents the average SNR and $R$ (or $\gamma$, indistinctly) denotes the instantaneous SNR. Since most performance metrics of interest for Rayleigh fading in the literature are usually given in closed-form ${ }^{2}$, the proposed approach allows for easily extending the results to Hoyt fading in a very simple manner. This is formally stated in the following lemma, where the Hoyt transform is introduced.

Lemma 2: Let $h(\gamma)$ be a performance metric depending on the instantaneous SNR $\gamma$, and let $\bar{h}_{R}(\bar{\gamma})$ be the metric in Rayleigh fading with average SNR $\bar{\gamma}$ obtained by averaging over an interval of the pdf of the SNR, i.e.,

$$
\bar{h}_{R}(\bar{\gamma})=\int_{a}^{b} h(x) \frac{1}{\bar{\gamma}} e^{-x / \bar{\gamma}} d x
$$

with $0 \leq a<b \leq \infty$. Then, the performance metric in Hoyt fading channels with average SNR $\bar{\gamma}$, denoted as $\bar{h}_{H}(\bar{\gamma})$, can be calculated as

$$
\bar{h}_{H}(\bar{\gamma})=\frac{1}{\pi} \int_{0}^{\pi} \bar{h}_{R}(\gamma(\theta, q)) d \theta \triangleq \mathcal{H}\left\{\bar{h}_{R}(\bar{\gamma}) ; q\right\} .
$$

\footnotetext{
${ }^{2}$ Throughout this paper, we consider a closed-form solution to any expression which is given in terms of a finite number of well-known functions in the context of communication theory, and for which numerical implementations in commercial mathematical packages are widely available.
} 
where $\mathcal{H}\{\cdot ; q\}$ is the Hoyt transform operation.

Proof: The metric $\bar{h}_{H}(\bar{\gamma})$ is obtained as

$$
\bar{h}_{H}(\bar{\gamma})=\int_{a}^{b} h(x) f(x) d x .
$$

where $f(x)$ is the pdf of a squared Hoyt random variable given in (12). Thus, we can write

$$
\bar{h}_{H}(\bar{\gamma})=\int_{a}^{b} h(x) \frac{1}{\pi} \int_{0}^{\pi} \frac{1}{\gamma(\theta, q)} e^{-x / \gamma(\theta, q)} d \theta d x,
$$

and reversing the order of integration ${ }^{3}$ yields

$$
\bar{h}_{H}(\bar{\gamma})=\frac{1}{\pi} \int_{0}^{\pi}\left[\int_{a}^{b} h(x) \frac{1}{\gamma(\theta, q)} e^{-x / \gamma(\theta, q)} d x\right] d \theta .
$$

By recognizing that the integral between brackets is actually $\bar{h}_{R}(\gamma(\theta, q)),(17)$ is finally obtained.

We see that Lemma 2 provides a simple and direct way to analyze the performance of communication systems operating in Hoyt fading channels. In fact, some interesting dualities with the popular MGF approach to the error-rate performance analysis of digital communication systems over fading channels [21] can be inferred:

In the reference work by Simon and Alouini, error-rate expressions are obtained "in the form of a single integral with finite limits and an integrand composed of elementary functions, thus readily enabling numerical evaluation"; in our work, the Hoyt transform also facilitates the derivation of expressions of the same form, with the integrand being now directly the performance metric obtained in the Rayleigh case. However, while the MGF approach is applicable to obtain a specific performance metric (error-rate) in general fading channels; the Hoyt transform approach is applicable to obtain general performance metrics in a specific fading channel (Hoyt).

An interesting consequence of Lemma 2 is the following corollary.

Corollary 1: The MGF of a squared Hoyt random variable of average $\bar{\gamma}$ and shape parameter $q$ can be written as

$$
\phi(s)=\frac{1}{\pi} \int_{0}^{\pi} \frac{1}{1-\gamma(\theta, q) s} d \theta .
$$

Proof: This result follows directly from Lemma 2 and the fact that the MGF of an exponentially distributed random variable of average $\bar{\gamma}$ is given by $(1-\bar{\gamma} s)^{-1}$.

This corollary provides an alternative demonstration of the integral representation of the pdf of a squared Hoyt random variable given in (12). Indeed, because of the way it has been constructed, it is clear that (21) is the MGF of a random variable which pdf is given by (12). On the other hand, the integral in (21) can be solved in closed-form, using [29, eq. (3.613.1)], yielding

$$
\phi(s)=\left[1-2 \bar{\gamma} s+\frac{q^{2}(2 \bar{\gamma} s)^{2}}{\left(1+q^{2}\right)^{2}}\right]^{-1 / 2},
$$

\footnotetext{
${ }^{3} \mathrm{~A}$ sufficient condition for the double integral to be reversible is that $h(x)$ is a nonnegative continuous function, which is the case of most performance metrics of interest, such as channel capacity, symbol error rate, outage probability, etc.
}

which is the well-known MGF of a squared Hoyt random variable [11]. Therefore, from the uniqueness theorem of the MGF, (1) and (12) are actually the same pdf.

Another benefit of the Hoyt transform method relies in the fact that the calculations are based on an integration involving a bounded trigonometric function; hence, this permits to find simple upper and lower bounds of the performance metrics. These bounds can be found by taking into account that symbol error rate performance metrics are usually convex decreasing functions with respect to the SNR, whereas channel capacity metrics are typically concave increasing functions. The following proposition establishes a sufficient condition to determine the monotonicity and convexity of some important average performance metric functions.

Proposition 2: Let $h(\gamma)$ be a performance metric depending on the instantaneous SNR $\gamma$ and let $\bar{h}_{R}(\bar{\gamma})$ be defined as in Lemma 1. If $h(\gamma)$ is a decreasing convex (increasing concave) function of $\gamma$ in $[0, \infty)$, then $\bar{h}_{R}(\bar{\gamma})$ is a decreasing convex (increasing concave) function of $\bar{\gamma}$.

Proof: If $h(\gamma)$ is a decreasing convex function then the first and second order derivatives of $h(\gamma)$ verify $h^{\prime}(\gamma) \leq 0$, $h^{\prime \prime}(\gamma) \geq 0$. By a simple change of variables in (16), considering the interval $[0, \infty)$, we can write

$$
\bar{h}_{R}(\bar{\gamma})=\int_{0}^{\infty} h(\bar{\gamma} x) e^{-x} d x,
$$

and its first and second order derivatives verify

$$
\begin{gathered}
\bar{h}_{R}^{\prime}(\bar{\gamma})=\int_{0}^{\infty} h^{\prime}(\bar{\gamma} x) x e^{-x} d x<0, \\
\bar{h}_{R}^{\prime \prime}(\bar{\gamma})=\int_{0}^{\infty} h^{\prime \prime}(\bar{\gamma} x) x^{2} e^{-x} d x>0 .
\end{gathered}
$$

Therefore, $\bar{h}_{R}(\bar{\gamma})$ is a decreasing convex function of $\bar{\gamma}$.

Analogously, if $h(\gamma)$ is an increasing concave function, then the first and second order derivatives of $h(\gamma)$ verify $h^{\prime}(\gamma) \geq 0$, $h^{\prime \prime}(\gamma) \leq 0$. Thus, $\bar{h}_{R}(\bar{\gamma})$ is an increasing concave function.

Now we present the aforementioned bounds in the next lemmas:

Lemma 3: Let $\bar{h}_{R}(\bar{\gamma})$ and $\bar{h}_{H}(\bar{\gamma})$ be functions obtained by averaging a given function $h(\gamma)$ in, respectively, Rayleigh and Hoyt fading channels, where $\bar{\gamma}$ is the average SNR, and let $\bar{h}_{R}(\bar{\gamma})$ be a decreasing convex function. Then, the following inequality holds:

$$
\bar{h}_{R}(\bar{\gamma}) \leq \bar{h}_{H}(\bar{\gamma}) \leq \bar{h}_{R}\left(\frac{2 q^{2}}{1+q^{2}} \bar{\gamma}\right)
$$

Lemma 4: Let $\bar{h}_{R}(\bar{\gamma})$ and $\bar{h}_{H}(\bar{\gamma})$ be functions obtained by averaging a given function $h(\gamma)$ in, respectively, Rayleigh and Hoyt fading channels, where $\bar{\gamma}$ is the average SNR, and let $\bar{h}_{R}(\bar{\gamma})$ be a concave increasing function. Then, the following inequality holds:

$$
\bar{h}_{R}\left(\frac{2 q^{2}}{1+q^{2}} \bar{\gamma}\right) \leq \bar{h}_{H}(\bar{\gamma}) \leq \bar{h}_{R}(\bar{\gamma}) .
$$

Proof: Let us first demonstrate (26): As $\bar{h}_{R}(\bar{\gamma})$ is a decreasing function of $\bar{\gamma}$ and the lowest value of $\gamma(\theta, q)$ is 
obtained for $\theta=0$, an upper bound of $\bar{h}_{H}(\bar{\gamma})$ can be found as

$$
\begin{aligned}
\bar{h}_{H}(\bar{\gamma})= & \frac{1}{\pi} \int_{0}^{\pi} \bar{h}_{R}(\gamma(\theta, q)) d \theta \leq \frac{1}{\pi} \int_{0}^{\pi} \bar{h}_{R}(\gamma(0, q)) d \theta \\
& =\bar{h}_{R}(\gamma(0, q))=\bar{h}_{R}\left(\frac{2 q^{2}}{1+q^{2}} \bar{\gamma}\right) .
\end{aligned}
$$

A lower bound of $\bar{h}_{H}(\bar{\gamma})$ can be found from Jensen's inequality and taking into account that $\bar{h}_{R}(\bar{\gamma})$ is convex:

$$
\begin{aligned}
\bar{h}_{R}(\bar{\gamma})= & \bar{h}_{R}\left(\frac{1}{\pi} \int_{0}^{\pi} \gamma(\theta, q) d \theta\right) \leq \\
& \frac{1}{\pi} \int_{0}^{\pi} \bar{h}_{R}(\gamma(\theta, q)) d \theta=\bar{h}_{H}(\bar{\gamma})
\end{aligned}
$$

On the other hand, (27) can be obtained analogously when $\bar{h}_{R}(\bar{\gamma})$ is a concave increasing function.

The bounds in Lemmas 3 and 4 state that performance in Hoyt fading, for a given average SNR, will be bounded between that of Rayleigh fading with the same average SNR and that of Rayleigh fading when the average SNR is scaled by a factor $2 q^{2} /\left(1+q^{2}\right)$. Note also that the derived bounds are asymptotically exact as $q \rightarrow 1$.

We have introduced a general approach to the analysis of wireless communication systems operating under Hoyt fading. In the following sections, we use this technique to derive novel results for different performance metrics of interest.

\section{Channel CAPACITY}

The channel capacity in Rayleigh fading channels was characterized in [12] for different transmission policies. Even though closed-form expressions were attained for the Rayleigh case, the channel capacity in Hoyt fading channels is much more complicated to evaluate. In fact, only infinite series expressions of very complicated argument are available in the literature $[14,15]$, which do not facilitate the extraction of any insights. Using the Hoyt transform method, we will now show how to use readily available performance results derived for Rayleigh channels to directly obtain the same performance metric in Hoyt fading.

The capacity per bandwidth unit using optimum rate adaptation (ORA) policy with constant transmit power is calculated as

$$
\frac{C_{\text {ora }}}{B}=\bar{C}=\int_{0}^{\infty} \log _{2}(1+\gamma) f_{\gamma}(\gamma) d \gamma .
$$

This capacity metric is obtained by averaging the Shannon capacity on a flat-fading channel using the pdf of $\gamma$, and has dimensions of bps/Hz. For a communication system operating under Rayleigh fading with average SNR at the receiver side given by $\bar{\gamma}$, a closed-form expression for this metric was obtained in [12, eq. 34] as

$$
\bar{C}^{\text {Ray }}=\log _{2}(e) e^{1 / \bar{\gamma}} E_{1}(1 / \bar{\gamma})
$$

where $E_{1}(\cdot)$ is the exponential integral function. Since $\bar{C}^{\text {Ray }}$ is computed in the form stated in Lemma 2, then we can directly calculate this metric considering a Hoyt fading channel as

$$
\bar{C}^{\text {Hoyt }}=\frac{\log _{2}(e)}{\pi} \int_{0}^{\pi} e^{1 / \gamma(\theta, q)} E_{1}\left(\frac{1}{\gamma(\theta, q)}\right) d \theta .
$$

Note that (32) is given in terms of a finite integral over a smooth and well-behaved function. Hence, it can be calculated accurately. A simple lower bound can be found as

$$
\bar{C}^{\text {Hoyt }} \geq \frac{1}{\ln 2} e^{\left(1+q^{2}\right) /\left(2 q^{2} \bar{\gamma}\right)} E_{1}\left(\frac{\left(1+q^{2}\right)}{2 q^{2} \bar{\gamma}}\right) .
$$

We now provide asymptotic capacity results in the low-SNR and high-SNR regimes. In the first situation, it is known that the asymptotic capacity in Rayleigh fading is given by [32]

$$
\left.\bar{C}_{\bar{\gamma} \Downarrow} \approx \log _{2} e \frac{d \phi(s)}{d s}\right|_{s=0}=\log _{2} e \cdot \bar{\gamma},
$$

where $\phi(s)$ represents the MGF of the SNR. Since (34) is obtained through linear operations over the distribution of the SNR, we can calculate the asymptotic capacity in Hoyt fading channels in the low-SNR regime as

$$
\bar{C}_{\bar{\gamma} \Downarrow}^{\text {Hoyt }} \approx \frac{\log _{2} e}{\pi} \int_{0}^{\pi} \gamma(\theta, q) d \theta=\log _{2} e \cdot \bar{\gamma},
$$

which is the same as in the Rayleigh case, but also the same as in the Rician case [33].

In the high-SNR regime, the asymptotic capacity can be expressed in the following form [34]

$$
\left.\bar{C}_{\bar{\gamma} \Uparrow} \approx \log _{2} e \cdot \frac{\partial}{\partial n} \mathbb{E}\left[\gamma^{n}\right]\right|_{n=0} .
$$

which is asymptotically exact. After some manipulations, we can equivalently express (36) as

$$
\bar{C}_{\bar{\gamma} \Uparrow} \approx \log _{2}(e) \cdot \log \bar{\gamma}-\mu,
$$

where $\mu$ is a constant value independent of the average SNR, but dependent on the specific channel model. The AWGN case yields a value of $\mu=0$, which is usually taken as a reference. The effect of fading causes $\mu>0$ and therefore there is a non-zero capacity loss due to fading for a given value of $\bar{\gamma}$. In the case of Rayleigh fading, it is a well-known result that $\mu=\log _{2}(e) \cdot \gamma_{e}$, where $\gamma_{e}$ is the Euler-Mascheroni constant. Therefore, for a fixed value of $\bar{\gamma}$, the parameter $\mu$ can be regarded as the capacity loss with respect to the AWGN case, being $\mu_{\text {Ray }} \approx 0.83 \mathrm{bps} / \mathrm{Hz}$.

There has been a renewed interest in the research community on different fading models that allow for characterizing a more severe fading condition than Rayleigh fading [35, 36]. Different models, such as the Two-Ray [35] or Hoyt fading meet this condition. Very recently, the value of $\mu$ for the TwoRay fading channel was derived [33], yielding a capacity loss of $\mu_{\text {Two-Ray }}=1 \mathrm{bps} / \mathrm{Hz}$. This shows that the Two-Ray model is indeed more detrimental than Rayleigh fading, as it provokes a larger capacity loss. However, the value of $\mu$ for Hoyt fading is unknown in the literature to the best of our knowledge. Using Lemma 2 in (37), we have

$$
\bar{C}_{\bar{\gamma} \Uparrow}^{\text {Hoyt }} \approx \frac{\log _{2}(e)}{\pi} \int_{0}^{\pi} \log \left\{\bar{\gamma}\left(1-\frac{1-q^{2}}{1+q^{2}} \cos \theta\right)\right\} d \theta-\mu_{\text {Ray }} .
$$

After some straightforward manipulations, we have

$\bar{C}_{\bar{\gamma} \Uparrow}^{\text {Hoyt }} \approx \log _{2}(e) \log \bar{\gamma}-\mu_{\text {Ray }}-\log _{2}(e) \log \left\{\frac{2\left(1+q^{2}\right)}{(1+q)^{2}}\right\}$. 
Therefore, the capacity loss in Hoyt fading with respect to the AWGN case is given by

$$
\mu_{\text {Hoyt }}=\underbrace{\log _{2}(e) \gamma_{e}}_{\mu_{\text {Ray }}}+\log _{2}(e) \log \left\{\frac{2\left(1+q^{2}\right)}{(1+q)^{2}}\right\} \text {. }
$$

The second term in (40) can be regarded as the capacity loss with respect to the Rayleigh case, and therefore equals 0 if $q=1$. In the limit case of $q=0$, corresponding to the onesided Gaussian distribution, we have the larger capacity loss given by

$$
\left.\mu_{\text {Hoyt }}\right|_{q=0}=\log _{2}(e) \gamma_{e}+1 \approx 1.83 \mathrm{bps} / \mathrm{Hz} .
$$

Therefore, the capacity loss of Hoyt fading with respect to AWGN can be as large as $1 \mathrm{bps} / \mathrm{Hz}$ more than in the Rayleigh case. Note that the $\mu_{\text {Hoyt }}$ can also be regarded as the high-SNR power offset in 3-dB units [37]. This means that the average SNR required to achieve a certain capacity $\bar{C}$ in the highSNR regime under Hoyt fading is $\Delta \gamma(\mathrm{dB})$ larger than in the AWGN case, with

$$
\begin{aligned}
\Delta \gamma_{\text {Hoyt }} & =\underbrace{3 \log _{2}(e) \gamma_{e}}_{\Delta \gamma_{\text {Ray }}}+3 \log _{2}(e) \log \left\{\frac{2\left(1+q^{2}\right)}{(1+q)^{2}}\right\}(\mathrm{dB}) \\
& \approx 2.5+3 \log _{2}(e) \log \left\{\frac{2\left(1+q^{2}\right)}{(1+q)^{2}}\right\}(\mathrm{dB})
\end{aligned}
$$

When $q=0$, the capacity loss of $1.83 \mathrm{bps} / \mathrm{Hz}$ is translated into a high-SNR power offset of about $5.5 \mathrm{~dB}$.

\section{Outage Probability of Secrecy Capacity}

\section{A. Problem Definition}

We consider the problem in which two legitimate peers, say Alice and Bob, wish to communicate over a wireless link in the presence of an eavesdropper, say Eve, that observes their transmission through a different link. Let us denote as $\gamma_{b}$ the instantaneous SNR at the receiver for the link between Alice and Bob, and let $\gamma_{e}$ be the instantaneous SNR at the eavesdropper for the wiretap link between Alice and Eve. We will assume $\gamma_{b}$ and $\gamma_{e}$ to be statistically independent.

Unlike the classical setup for the Gaussian wiretap channel [38], it is known that fading provides an additional layer of security to the communication between Alice and Bob [22, 39], allowing for a secure transmission even when Eve experiences a better SNR than the legitimate receiver Bob.

According to the information-theoretic formulation in [22], the secrecy capacity in this scenario is defined as

$$
C_{S}=\left[C_{B}-C_{E}\right]^{+},
$$

where $[a]^{+} \equiv \max \{a, 0\}, C_{B}$ is the capacity of the main channel

$$
C_{B}=\log \left(1+\gamma_{b}\right),
$$

and $C_{E}$ is the capacity of the eavesdropper channel

$$
C_{E}=\log \left(1+\gamma_{e}\right) .
$$

For the sake of simplicity, we assumed a normalized bandwidth $B=1$ in the previous capacity definitions.
In [22, 39], the physical layer security of the communication between Alice and Bob in the presence of Eve was characterized in terms of several performance metrics of interest, assuming that both wireless links undergo Rayleigh fading. Specifically, closed-form expressions were derived for the probability of strictly positive secrecy capacity $\mathcal{P}\left(C_{S}>0\right)$, and for the outage probability of the secrecy capacity $\mathcal{P}\left(C_{S}<R_{S}\right)$, where $R_{S}$ is defined as the threshold rate under which secure communication cannot be achieved. As these expressions will be used in the forthcoming analysis, we reproduce them for the readers' convenience

$$
\begin{aligned}
\mathcal{P}\left(C_{S}>0\right) & =\frac{\bar{\gamma}_{b}}{\bar{\gamma}_{b}+\bar{\gamma}_{e}}, \\
\mathcal{P}\left(C_{S}<R_{S}\right) & =1-\frac{\bar{\gamma}_{b}}{\bar{\gamma}_{b}+2^{R_{S}} \bar{\gamma}_{e}} \exp \left(-\frac{2^{R_{S}}-1}{\bar{\gamma}_{b}}\right),
\end{aligned}
$$

where $\bar{\gamma}_{b}$ and $\bar{\gamma}_{e}$ are the average SNRs at Bob and Eve, respectively.

We note that $\mathcal{P}\left(C_{S}>0\right)=1-\mathcal{P}\left(C_{S}<R_{S}\right)_{R_{S}=0}$; hence, the probability of strictly positive secrecy capacity will be considered as a particular case of the secrecy outage probability.

\section{B. Secrecy Outage Probability Analysis}

Let us consider the scenario where the wireless links experience a more severe fading than Rayleigh, say Hoyt, where $q_{b}$ and $q_{e}$ represent the Hoyt shape parameters for the desired and eavesdropper links, respectively. We also define the eccentricities associated with both Hoyt distributions as $\epsilon_{b}=\frac{1-q_{b}^{2}}{1+q_{b}^{2}}$ and $\epsilon_{e}=\frac{1-q_{e}^{2}}{1+q_{e}^{2}}$

According to the Hoyt transform, the outage probability of the secrecy capacity in Hoyt fading channels is given by

$$
\begin{aligned}
\mathcal{P}\left(C_{S}<\right. & \left.R_{S}\right)=1-\frac{1}{\pi^{2}} \int_{0}^{\pi} \int_{0}^{\pi} \exp \left(-\frac{2^{R_{S}-1}}{\bar{\gamma}_{b}\left(1-\epsilon_{b} \cos \theta_{b}\right)}\right) \\
& \times \frac{\bar{\gamma}_{b}\left(1-\epsilon_{b} \cos \theta_{b}\right)}{\bar{\gamma}_{b}\left(1-\epsilon_{b} \cos \theta_{b}\right)+2^{R} S \bar{\gamma}_{e}\left(1-\epsilon_{e} \cos \theta_{e}\right)} d \theta_{e} d \theta_{b} .
\end{aligned}
$$

We observe that the integral over $\theta_{e}$ can be solved, and hence we obtain

$$
\begin{aligned}
P\left(C_{S}<\right. & \left.R_{S}\right)=1-\frac{1}{\pi} \int_{0}^{\pi} \exp \left(-\frac{2^{R} S-1}{\bar{\gamma}_{b}(\theta)}\right) \\
& \times \frac{\bar{\gamma}_{b}(\theta)}{\bar{\gamma}_{b}(\theta)+2^{R_{S}} \bar{\gamma}_{e}} \frac{1}{\sqrt{1-\left(\frac{\epsilon_{e} 2^{R} \bar{\gamma}_{e}}{\bar{\gamma}_{b}(\theta)+2^{R} S \bar{\gamma}_{e}}\right)^{2}}} d \theta
\end{aligned}
$$

where $\bar{\gamma}_{b}(\theta)=\bar{\gamma}_{b}\left(1-\epsilon_{b} \cos \theta\right)$. Hence, the secrecy capacity OP is given in terms of a simple integral form. This result is new in the literature to the best of our knowledge, and shows the strength and versatility of the Hoyt transform method to derive new performance metrics for Hoyt fading by leveraging existing results for Rayleigh fading.

Directly from (50), the probability of strictly positive secrecy capacity can be easily obtained as

$$
P\left(C_{S}>0\right)=\frac{1}{\pi} \int_{0}^{\pi} \frac{\bar{\gamma}_{b}(\theta)}{\bar{\gamma}_{b}(\theta)+\bar{\gamma}_{e}} \frac{1}{\sqrt{1-\left(\frac{\varepsilon_{e} \bar{\gamma}_{e}}{\bar{\gamma}_{b}(\theta)+\bar{\gamma}_{e}}\right)^{2}}} d \theta .
$$

Expressions (50) and (51) admit an easy manipulation, in order to extract insights on the effect of fading severity into the secrecy capacity OP. One clear example arises if we 
assume that the eavesdropper link suffers from a more severe fading compared to the desired link: this can be achieved by setting $q_{b}=1$, and seeing what is the impact of $q_{e}$. In this case, the integral over $\theta_{b}$ disappears, yielding to a closedform expression for the secrecy capacity $\mathrm{OP}$, and hence for $P\left(C_{S}>0\right)$ :

$$
\left.P\left(C_{S}<R_{S}\right)\right|_{q_{b}=1}=1-\frac{\bar{\gamma}_{b}}{\bar{\gamma}_{b}+2^{R_{S}} \bar{\gamma}_{e}} \frac{\exp \left(-\frac{2^{R_{S}}-1}{\bar{\gamma}_{b}}\right)}{\sqrt{1-\left(\frac{\varepsilon_{e} 2^{R_{S}} \bar{\gamma}_{e}}{\bar{\gamma}_{b}+2^{R} \bar{\gamma}_{e}}\right)^{2}}} .
$$

Comparing (48) and (52), we observe that both have similar form, and the effect of the distribution of the fading for the eavesdropper link is captured by a multiplicative term that modulates the result for the Rayleigh case. Since this additional term is always larger than one, it is clear that for a fixed value of $\bar{\gamma}_{b}$ and $\bar{\gamma}_{e}$, the secrecy capacity OP $P\left(C_{S}<R_{S}\right)$ decreases with $q_{e}$. This illustrates the fact that when Eve suffers from a more severe fading, then the probability of having a secure communication between Alice and Bob grows.

A similar conclusion can be extracted when examining the probability of strictly positive secrecy capacity in this particular scenario:

$$
\left.P\left(C_{S}>0\right)\right|_{q_{b}=1}=\frac{\bar{\gamma}_{b}}{\bar{\gamma}_{b}+\bar{\gamma}_{e}} \frac{1}{\sqrt{1-\left(\frac{\epsilon_{e} \bar{\gamma}_{e}}{\bar{\gamma}_{b}+\bar{\gamma}_{e}}\right)^{2}}} .
$$

Again, the effect of considering $q_{e}<1$ (i.e. a more severe fading than Rayleigh for the eavesdropper link) causes that $P\left(C_{S}>0\right)$ grows as $q_{e}$ is reduced.

\section{Asymptotic Analysis}

An asymptotic analysis is now performed of the high-SNR regime for the outage probability of secrecy capacity, and of the low-SNR regime for the probability of strictly positive secrecy capacity, as meaningful insights can be obtained in these cases.

According to the expression in (48) and letting $\bar{\gamma}_{b} \Uparrow$, we have that the outage probability of the secrecy capacity in Rayleigh fading channels can be approximated by

$$
\mathcal{P}\left(C_{S}<R_{S}\right)_{\bar{\gamma}_{b} \Uparrow}^{\text {Ray }} \approx \frac{2^{R_{S}} \bar{\gamma}_{e}+2^{R_{S}}-1}{\bar{\gamma}_{b}} .
$$

Now, direct application of the Hoyt transform yields the asymptotic outage probability of the secrecy capacity in Hoyt fading channels as

$$
\begin{aligned}
\mathcal{P}( & \left.C_{S}<R_{S}\right)_{\bar{\gamma}_{b} \Uparrow}^{\text {Hoyt }} \approx \frac{1}{\pi^{2}} \\
& \times \int_{0}^{\pi} \int_{0}^{\pi} \frac{2^{R_{S}} \bar{\gamma}_{e}\left(1+\epsilon_{e} \cos \theta_{e}\right)+2^{R_{S}}-1}{\bar{\gamma}_{b}\left(1+\epsilon_{b} \cos \theta_{b}\right)} d \theta_{e} d \theta_{b} \\
& \approx \frac{2^{R_{S}} \bar{\gamma}_{e}+2^{R_{S}}-1}{\bar{\gamma}_{b}} \frac{1}{\sqrt{1-\epsilon_{b}^{2}}} .
\end{aligned}
$$

Noteworthy, in the high-SNR regime the outage probability of the secrecy capacity does not depend on the fading severity of the eavesdropper channel. Instead, the effect of the eavesdropper is only determined by its average SNR $\bar{\gamma}_{e}$. Thus, in the
high-SNR regime the distribution of $\gamma_{b}$ has a dominant effect on this probability metric.

Let us now consider the low-SNR regime. In this situation, we have $\bar{\gamma}_{b} \ll \bar{\gamma}_{e}$ and in the absence of fading the secrecy capacity equals zero. However, because of the effect of the assumed independent fading at the desired and the eavesdropper's channels, it is possible to achieve a secure communication with a certain probability. The asymptotic value of the probability of strictly positive secrecy capacity in the low-SNR regime under Rayleigh fading can be easily derived from (47), yielding

$$
\mathcal{P}\left(C_{S}>0\right)_{\bar{\gamma}_{b} \Downarrow}^{\text {Ray }} \approx \frac{\bar{\gamma}_{b}}{\bar{\gamma}_{e}} .
$$

Using again the Hoyt transform over (57) yields the desired asymptotic value for the Hoyt case as

$$
\begin{aligned}
\mathcal{P}\left(C_{S}>0\right)_{\bar{\gamma}_{b} \Downarrow}^{\text {Hoyt }} & \approx \frac{1}{\pi^{2}} \int_{0}^{\pi} \int_{0}^{\pi} \frac{\bar{\gamma}_{b}\left(1+\epsilon_{b} \cos \theta_{b}\right)}{\bar{\gamma}_{e}\left(1+\epsilon_{e} \cos \theta_{e}\right)} d \theta_{e} d \theta_{b} \\
& \approx \frac{\bar{\gamma}_{b}}{\bar{\gamma}_{e}} \frac{1}{\sqrt{1-\epsilon_{e}^{2}}} .
\end{aligned}
$$

We now observe that (59) is independent of $\epsilon_{b}$. It is now the fading severity of the eavesdropper channel the one that has a dominant effect over the probability of strictly positive secrecy capacity in the low-SNR regime, whereas the effect of Bob's channel is only incorporated through $\bar{\gamma}_{b}$.

\section{NUMERICAL RESULTS}

After describing how the Hoyt transform method can be applied to obtain new results for some information and communication-theoretic performance metrics, now we discuss the main implications that arise in practical scenarios.

\section{A. Channel capacity}

First, we study the Shannon capacity of ORA technique in Hoyt fading channels. In the following set of figures, we evaluate the capacity per bandwidth unit in Hoyt fading channels using (32).

In Fig. 1, we observe how the capacity loss due to a more severe fading is low for values of $q>0.5$, being under 0.15 $\mathrm{bps} / \mathrm{Hz}$ in this range. In fact, it is noted how the achievable performance when $q=0.9$ is practically coincident with the Rayleigh case. Markers indicate the simple lower bounds obtained in (33). We see how the lower bound becomes tighter as $q$ is increased, whereas the performance for the Rayleigh case serves as an upper bound.

The accuracy of the asymptotic approximations yielding from (36) in the high-SNR regime is evaluated in Fig. 2. The capacity of the AWGN and Rayleigh $(q=1)$ cases are included as reference.

We observe that for values of $q$ close to 1 , the capacity loss with respect to the Rayleigh case is almost negligible. However, we see that as $q$ is reduced, the gap between the capacities in the Rayleigh and Hoyt cases grows. We see how the approximations for the capacity are asymptotically exact for sufficiently large $\bar{\gamma}$. However, we also observe that for 


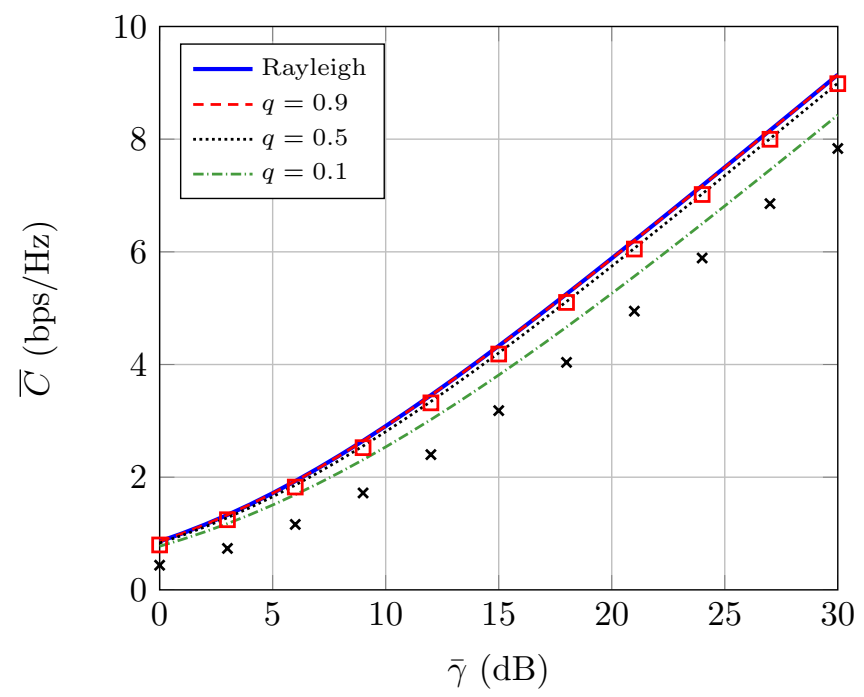

Figure 1. Normalized capacity $\bar{C}$ vs. $\bar{\gamma}$ using ORA policy, for different values of $q$. Markers indicate the lower bounds on capacity given by (33), for $q=0.5$ ('x') and $q=0.9$ (squares).

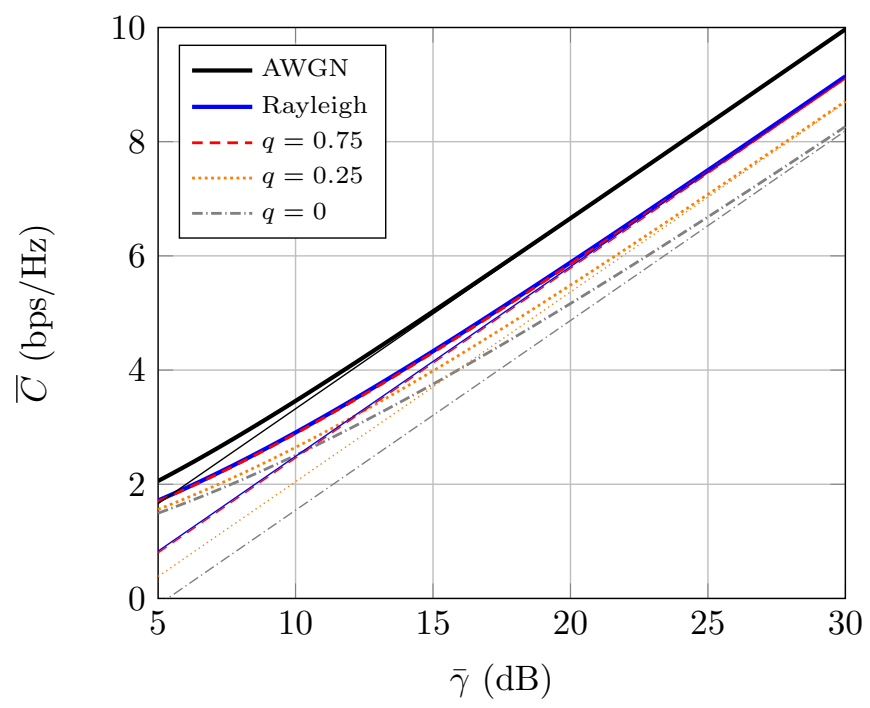

Figure 2. Normalized capacity $\bar{C}$ vs. $\bar{\gamma}$ using ORA policy, for different values of $q$. Straight thin lines represent the asymptotic results for high-SNR.

$q=0$ the convergence between the exact and asymptotic capacity takes place at a larger value of $\bar{\gamma}$. We must note that the asymptotic results obtained for Hoyt fading are also valid for communication systems using optimal power and rate adaptation (OPRA) policy, since in the high-SNR regime this policy has the same performance as ORA [12].

In Fig. 3, we study the asymptotic capacity loss due to Hoyt fading with respect to the reference AWGN case, which is given by (40). The capacity loss for Rayleigh and Two-Ray [33] are also included for comparison purposes. As indicated in (41), the maximum capacity loss is obtained when $q=0$. This corresponds to the more severe fading condition that Hoyt fading can represent. As $q \rightarrow 1$, we see that the capacity loss tends to the value obtained in [12], i.e. approximately 0.83 bps/Hz. The comparison with the Two-Ray fading model is

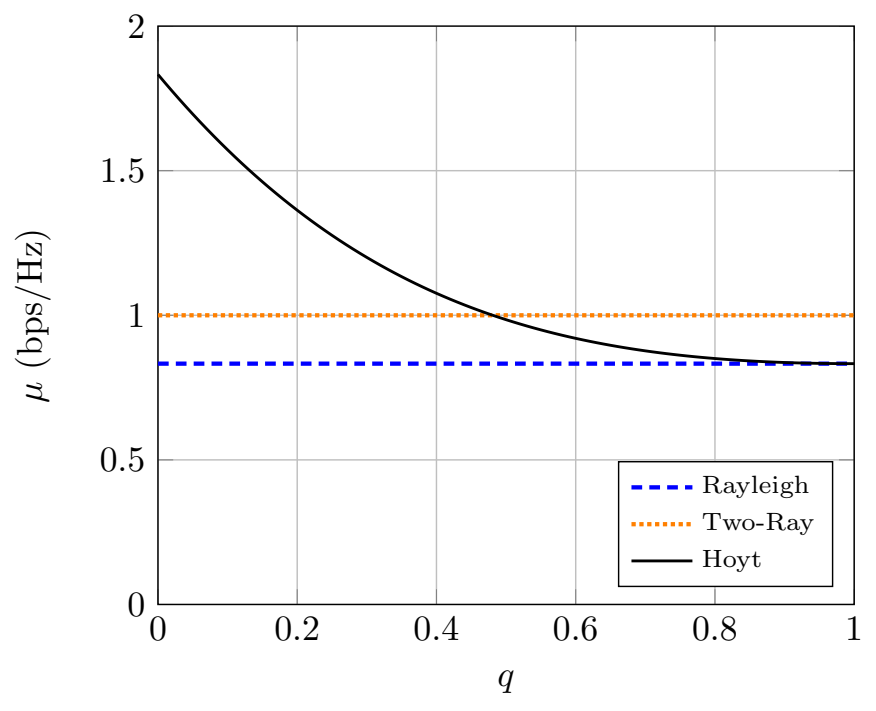

Figure 3. Asymptotic capacity loss $\mu_{\mathrm{Hoyt}}$ in (40) as a function of $q$.

also interesting: we see that the capacity loss in Hoyt and Two-Ray fading is coincident for $q \approx 0.48$. This suggests that Hoyt fading represents a more severe condition than the TwoRay model if $q<0.48$, when the asymptotic capacity loss is chosen as the performance metric of interest.

\section{B. Secrecy capacity}

Now we focus on the scenario considered in Section V; specifically, we will evaluate the effect of considering that the links between Alice and Bob (and equivalently between Alice and Eve) can suffer from different fading severities, quantified by the parameters $q_{b}$ and $q_{e}$. In Fig. 4, the secrecy capacity OP derived in (50) is represented as a function of the average SNR at Bob $\bar{\gamma}_{b}$, for different sets of values of the Hoyt shape parameters. We assume that the normalized rate threshold value used to declare an outage is $R_{S}=0.1$, and an average SNR at Eve $\bar{\gamma}_{e}=15 \mathrm{~dB}$.

For a given value of $q_{b}$, we observe two different effects depending on the magnitude of $\bar{\gamma}_{b}$ : in the low-medium SNR region, we see how a lower value of $q_{e}$ (i.e. a more severe fading in the eavesdropper link) makes the occurrence of a secrecy outage to be less likely. Hence, $\mathcal{P}\left(C_{S}<R_{S}\right)$ decreases with $q_{e}$ for a given $\bar{\gamma}_{b}$; we also note how the secrecy in this region is barely affected by the value of $q_{b}$. Conversely, in the high-SNR region we observe how the outage secrecy probability is mainly dominated by the fading severity of the desired link $q_{b}$ as predicted by (56). In this region, it is the distribution of $\gamma_{b}$ the dominant factor in the secure communication between Alice and Bob.

The probability of strictly positive secrecy capacity given in (51) is evaluated in Fig. 5, for the same set of parameter values considered in the previous figure. We can extract similar conclusions with regard of the effects of the fading severity in the desired and eavesdropper links. For low values of $\bar{\gamma}_{b}$, the secure communication is mainly determined by the distribution of $\gamma_{e}$ as indicated in (59); specifically, considering $\bar{\gamma}_{b}=5 \mathrm{~dB}$ we see how $\mathcal{P}\left(C_{S}>0\right)$ is twice larger for $q_{e}=0.1$, compared 


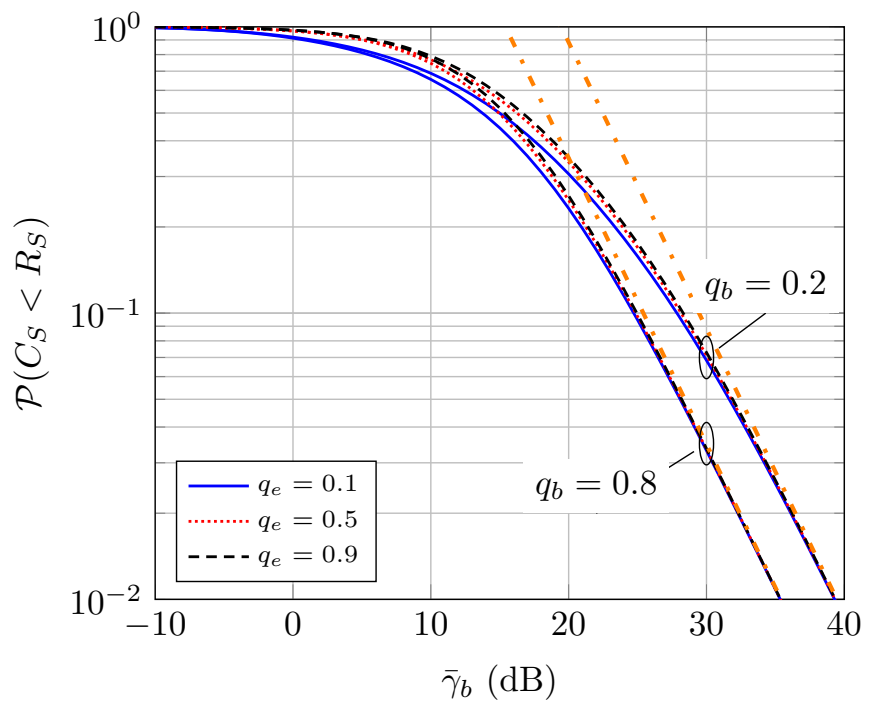

Figure 4. Outage probability of secrecy capacity as a function of $\bar{\gamma}_{b}$, for different values of $q_{e}$ and $q_{b}$. Parameter values $\bar{\gamma}_{e}=15 \mathrm{~dB}$ and $R_{S}=0.1$. Asymptotic values correspond to (56).

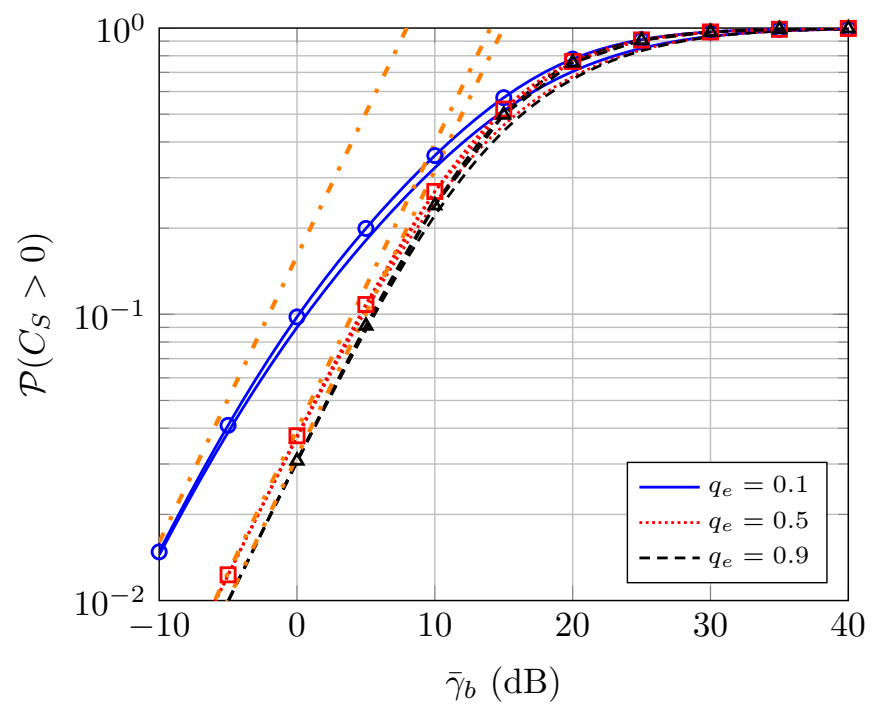

Figure 5. Probability of strictly positive secrecy capacity as a function of $\bar{\gamma}_{b}$, for different values of $q_{e}$ and $q_{b}$. Parameter value $\bar{\gamma}_{e}=15 \mathrm{~dB}$. Lines without markers indicate $q_{b}=0.2$; lines with markers are included for $q_{b}=0.8$. Asymptotic values correspond to (59).

to $q_{e}=0.5$. We also observe how a less severe fading in the desired link (i.e. a larger value of $q_{b}$ ) leads this probability to be larger.

\section{CONCLUSIONS}

We have provided a new look at the analysis of wireless communication systems in Hoyt (Nakagami- $q$ ) fading. Unlike previous approaches in the literature, we have found a connection between the Rayleigh and Hoyt distributions that facilitates the analysis in the latter scenario.

By deriving integral expressions for the pdf and cdf of the squared Hoyt distribution, we have shown that the squared Hoyt distribution is in fact a composition of exponential distributions with continuously varying averages. Using this connection, we have introduced the Hoyt transform approach as a way to obtain easy-to-compute finite-range integral expressions of different performance metrics in Hoyt fading channels, as well as simple upper and lower bounds which become asymptotically tight as $q \rightarrow 1$, by simply leveraging existing results for Rayleigh fading channels.

As a direct application, we have derived new expressions for several scenarios of interest in information and communication theory: (a) capacity analysis in Hoyt fading channels; and (b) wireless information-theoretic security in Hoyt fading.

A further implication of the results in this paper is that there is no need to reproduce complicated calculations for Hoyt fading in the cases where tractable expressions are available for the Rayleigh case. Instead, these analyses can be easily extended to Hoyt scenarios by using a straightforward finiterange integral.

\section{REFERENCES}

[1] P. Beckmann, Probability in communication engineering, ser. Harbrace series in electrical engineering. Harcourt, Brace \& World, 1967.

[2] S. O. Rice, "Mathematical analysis of random noise," Bell Syst. Tech. J., vol. 24, no. 1, pp. 46-156, Jan 1945.

[3] R. S. Hoyt, "Probability functions for the modulus and angle of the normal complex variate," Bell Syst. Tech. J., vol. 26, no. 2, pp. 318359, April 1947.

[4] V. Aalo, G. Efthymoglou, and C. Chayawan, "On the envelope and phase distributions for correlated Gaussian quadratures," IEEE Commun. Lett., vol. 11, no. 12, pp. 985-987, December 2007.

[5] P. Dharmawansa, N. Rajatheva, and C. Tellambura, "Envelope and phase distribution of two correlated Gaussian variables," IEEE Trans. Commun., vol. 57, no. 4, pp. 915-921, April 2009.

[6] B. Picinbono, "On circularity," IEEE Trans. Signal Process., vol. 42, no. 12, pp. 3473-3482, Dec 1994.

[7] P. Schreier, L. Scharf, and C. Mullis, "Detection and estimation of improper complex random signals," IEEE Trans. Inf. Theory, vol. 51, no. 1 , pp. 306-312, Jan 2005.

[8] M. Nakagami, "The m-distribution: A general formula of intensity distribution of rapid fading," Statistical Method of Radio Propagation, 1960.

[9] N. Youssef, C.-X. Wang, and M. Patzold, "A Study on the Second Order Statistics of Nakagami-Hoyt Mobile Fading Channels," IEEE Trans. Veh. Technol., vol. 54, no. 4, pp. 1259-1265, July 2005.

[10] J. F. Paris, "Nakagami-q (Hoyt) distribution function with applications," Electron. Lett., vol. 45, no. 4, pp. 210-211, February 2009.

[11] M. K. Simon and M.-S. Alouini, Digital communication over fading channels. Wiley-IEEE Press, 2005.

[12] M.-S. Alouini and A. J. Goldsmith, "Capacity of Rayleigh fading channels under different adaptive transmission and diversity-combining techniques," IEEE Trans. Veh. Technol., vol. 48, no. 4, pp. 1165-1181, 1999.

[13] A. Annamalai, C. Tellambura, and V. K. Bhargava, "Simple and accurate methods for outage analysis in cellular mobile radio systems-a unified approach," IEEE Trans. Commun., vol. 49, no. 2, pp. 303-316, Feb 2001.

[14] J. Cheng and T. Berger, "Capacity of Nakagami-q (Hoyt) fading channels with channel side information," in International Conference on Соттиnication Technology 2003, April 2003, pp. 1915-1918.

[15] R. Subadar and P. Sahu, "Channel capacity of adaptive transmission with L-MRC systems in Hoyt (Nakagami-q) fading channels," in 2010 Annual IEEE India Conference (INDICON), Dec 2010, pp. 1-4.

[16] S. Khatalin and J. P. Fonseka, "On the channel capacity in Rician and Hoyt fading environments with MRC diversity," IEEE Trans. Veh. Technol., vol. 55, no. 1, pp. 137-141, Jan 2006.

[17] J. F. Paris and D. Morales-Jimenez, "Outage probability analysis for Nakagami-q (Hoyt) fading channels under Rayleigh interference," IEEE Trans. Wireless Commun., vol. 9, no. 4, pp. 1272-1276, April 2010.

[18] F. J. Lopez-Martinez, R. F. Pawula, E. Martos-Naya, and J. F. Paris, “A Clarification of the Proper-Integral Form for the Gaussian Q-Function and Some New Results Involving the F-Function," IEEE Commun. Lett., vol. 18, no. 9, pp. 1495-1498, Sept 2014. 
[19] M. K. Simon, "A new twist on the Marcum Q-function and its application," IEEE Commun. Lett., vol. 2, no. 2, pp. 39-41, Feb 1998.

[20] R. F. Pawula, S. O. Rice, and J. Roberts, "Distribution of the Phase Angle Between Two Vectors Perturbed by Gaussian Noise," IEEE Trans. Commun., vol. 30, no. 8, pp. 1828-1841, Aug 1982.

[21] M. K. Simon and M.-S. Alouini, "A unified approach to the performance analysis of digital communication over generalized fading channels," Proceedings of the IEEE, vol. 86, no. 9, pp. 1860-1877, Sep 1998.

[22] J. Barros and M. R. D. Rodrigues, "Secrecy Capacity of Wireless Channels," in IEEE International Symposium on Information Theory, 2006, July 2006, pp. 356-360.

[23] M. Z. I. Sarkar, T. Ratnarajah, and M. Sellathurai, "Secrecy capacity of Nakagami-m fading wireless channels in the presence of multiple eavesdroppers," in 43rd Asilomar Conference on Signals, Systems and Computers, Nov 2009, pp. 829-833.

[24] X. Liu, "Probability of strictly positive secrecy capacity of the ricianrician fading channel," IEEE Wireless Commun. Lett., vol. 2, no. 1, pp. 50-53, February 2013.

[25] L. Wang, N. Yang, M. Elkashlan, P. L. Yeoh, and J. Yuan, "Physical Layer Security of Maximal Ratio Combining in Two-Wave With Diffuse Power Fading Channels," IEEE Trans. Inf. Forensics Security, vol. 9, no. 2, pp. 247-258, Feb 2014.

[26] B. Tan, T. Tjhung, C. Teo, and P. Leong, "Series Representations for Rice's Ie Function," IEEE Trans. Commun., vol. 32, no. 12, pp. 1342 1344, Dec 1984

[27] P. Sofotasios and S. Freear, "Analytic expressions for the Rice Iefunction and the incomplete Lipschitz-Hankel Integrals," in Annиal IEEE India Conference (INDICON) 2011, Dec 2011, pp. 1-6.

[28] R. F. Pawula, "Relations between Rice Ie-function and Marcum Qfunction with applications to error rate calculations," Electron. Lett., vol. 31, no. 20, pp. 1717-1719, Sep 1995.

[29] I. S. Gradshteyn and I. M. Ryzhik, Table of Integrals, Series and Products, 7th ed. Academic Press Inc, 2007. [Online]. Available: http://www.worldcat.org/isbn/012294755X

[30] R. F. Pawula, "A new formula for MDPSK symbol error probability," IEEE Commun. Lett., vol. 2, no. 10, pp. 271-272, Oct 1998.

[31] A. Coluccia, "On the Expected Value and Higher-Order Moments of the Euclidean Norm for Elliptical Normal Variates," IEEE Commun. Lett., vol. 17, no. 12, pp. 2364-2367, December 2013.

[32] M. Di Renzo, F. Graziosi, and F. Santucci, "Channel capacity over generalized fading channels: A novel MGF-based approach for performance analysis and design of wireless communication systems," IEEE Trans. Veh. Technol., vol. 59, no. 1, pp. 127-149, 2010.

[33] M. Rao, F. J. Lopez-Martinez, M.-S. Alouini, and A. Goldsmith, "MGF Approach to the Analysis of Generalized Two-Ray Fading Models," IEEE Trans. Wireless Commun., vol. 14, no. 5, pp. 2548-2561, May 2015.

[34] F. Yilmaz and M.-S. Alouini, "A unified MGF-based capacity analysis of diversity combiners over generalized fading channels," IEEE Trans. Commun., vol. 60, no. 3, pp. 862-875, 2012.

[35] J. Frolik, "On appropriate models for characterizing hyper-Rayleigh fading," IEEE Trans. Wireless Commun., vol. 7, no. 12, pp. 5202-5207, 2008.
[36] D. Matolak and J. Frolik, "Worse-than-Rayleigh fading: Experimental results and theoretical models," IEEE Commun. Mag., vol. 49, no. 4, pp. 140-146, April 2011.

[37] A. Lozano, A. M. Tulino, and S. Verdu, "High-SNR power offset in multiantenna communication," IEEE Trans. Inf. Theory, vol. 51, no. 12, pp. 4134-4151, Dec 2005.

[38] S. Leung-Yan-Cheong and M. E. Hellman, "The Gaussian wire-tap channel," IEEE Trans. Inf. Theory, vol. 24, no. 4, pp. 451-456, Jul 1978.

[39] M. Bloch, J. Barros, M. R. D. Rodrigues, and S. W. McLaughlin, "Wireless Information-Theoretic Security," IEEE Trans. Inf. Theory, vol. 54, no. 6, pp. 2515-2534, June 2008

Juan M. Romero-Jerez holds MSc degrees both in Telecommunication Engineering and in Mathematics from the University of Malaga, Spain, where he also obtained a $\mathrm{PhD}$ degree in Telecommunication Engineering in 2001. In 1996 he joined the Electronic Technology Department at the University of Malaga, where he is an Associate Professor since 2002. He was a Visiting Associate Professor in the Electrical Engineering Department at Stanford University from September 2005 to February 2006, and also from September 2007 to February 2008, and from January 2016 to February 2016.

$\mathrm{He}$ has participated in several research projects in the areas of packet radio transmission, multiple antennas, interference management and cellular networks. His current research interest is in the area of wireless communications and, more specifically: wireless communications performance analysis, multipath fading, wireless channel modeling, diversity systems, smart antennas, MIMO performance and interference management. He is a Senior Member of the IEEE, and an Editor of the IEEE Transactions on Wireless Communications.

F. Javier Lopez-Martinez received the M.Sc. and Ph.D. degrees in Telecommunication Engineering in 2005 and 2010, respectively, from the University of Malaga (Spain). He joined the Communication Engineering Department at the University of Malaga in 2005 as an associate researcher. In 2010 he stayed for 3 months as a visitor researcher at University College London. He was a Marie Curie postdoctoral fellow in the Wireless Systems Lab at Stanford University (2012-2014), and at University of Malaga (2014-2015). Since 2015, he is an Assistant Professor at the Communication Engineering Department, University of Malaga.

He has received several research awards, including the best paper award in the Communication Theory symposium at IEEE Globecom 2013, the IEEE Communications Letters Exemplary Reviewer certificate in 2014, and the IEEE Transactions on Communications Exemplary Reviewer certificate in 2015. His research interests span a diverse set of topics in the wide areas of Communication Theory and Wireless Communications: stochastic processes, wireless channel modeling, random matrix theory, physical layer security, massive MIMO and mmWave for $5 \mathrm{G}$ 